\title{
Caractéristiques cinématiques et structurelles d'un jet d'air plan turbulent frappant une plaque plane placée à distance variable
}

\author{
Stéphane Maurel ${ }^{1}$, Claude Rey ${ }^{2}$, Camille Solliec ${ }^{3, a}$ Et Michel Pavageau ${ }^{3}$ \\ 1 Gaz de France, Direction de la Recherche, DU - MEI, 361 avenue du Président Wilson, BP 33, \\ 93211 Saint-Denis La Plaine Cedex, France \\ 2 IUT de Marseille, Département Thermique et Énergétique, 142 traverse Charles Susini, 13388 Marseille Cedex 13, France \\ 3 École des mines de Nantes, GEPEA UMR CNRS 6144, 4 rue A. Kastler, BP 20722, 44307 Nantes Cedex 3, France
}

Reçu le 28 mars 2003, accepté le 23 October 2003

\begin{abstract}
Résumé - Le développement du jet libre est relativement bien connu, ce n'est pas le cas du jet plan turbulent en présence d'un impact. En effet, selon la distance $H$ de la paroi d'impact, et de l'épaisseur initiale $e$ du jet, le développement se fera de manière relativement différente à cause du confinement qui conditionne l'expansion du jet. À la différence du jet libre, il n'existe pas à l'heure actuelle de base de données complète ni de modèle global permettant de décrire analytiquement la décroissance de la vitesse moyenne dans le plan de symétrie des jets plans en impact. La littérature fait bien état de lois semiempiriques, mais les relations correspondantes ne concernent que quelques zones de la région de jet : le cône potentiel et la zone d'affinité principalement. Le travail présenté concerne d'une part, l'établissement d'une corrélation générale capable de décrire l'évolution de la vitesse axiale quelles que soient l'épaisseur du jet et la distance à l'impact, et d'autre part, la description du champ de turbulence du jet et les lois d'épanouissement latéral. Les résultats du modèle de décroissance de la vitesse axiale sont aussi comparés et validés à l'aide de mesures expérimentales obtenues par vélocimétrie laser à effet Doppler.
\end{abstract}

Mots clés : Jet plan turbulent / impact / modèle empirique / LDV / PIV

Abstract - Cinematic and structural characteristics of a turbulent planar jet impinging a flat plate with variable distance. The development of the free jet is relatively well known, it is not the case of the impinging turbulent plane jet. Indeed, according both to the distance $H$ of the plate of impingement, and to the initial thickness $e$ of the jet, its development will be relatively different because the plate distance $\mathrm{H} / \mathrm{e}$ strongly conditions the expansion of the jet. Work presented deals principally with the development of a general correlation able to predict the decrease of the axial velocity whatever the thickness of the jet and the impinging distance. The results are compared and validated by using experimental measurements obtained by laser Doppler velocimetry. An other part of the paper describes the turbulence intensity field and the half width of the jet for different $H / e$ ratios.

Key words: Turbulent planar jet / impingement / empirical model / LDV / PIV

\section{Introduction}

Les dispositifs à jet d'air sont utilisés dans de nombreuses applications industrielles pour réchauffer, refroidir, nettoyer ou protéger certains produits en cours de fabrication (industries agroalimentaires, pharmaceutiques ou électroniques par exemple). En matière de protection rapprochée, le principe consiste à souffler un jet d'air propre sur la zone à protéger. Ce jet peut ainsi réduire voire inhiber les transferts de masse et de chaleur en

\footnotetext{
a Auteur correspondant : camille.solliec@emn.fr
}

provenance de l'extérieur de la zone à protéger ou au contraire augmenter les échanges.

Concernant le chauffage, le refroidissement ou le nettoyage, on cherchera à augmenter les transferts de masse ou de chaleur dans la zone d'impact. Dans ce cas, il s'agit de bien connaître les propriétés cinématiques (champ de vitesse, de turbulence et structure de l'écoulement) du jet dans la zone d'impact en fonction des dimensions du système [1].

D'autres applications se rencontrent dans le secteur du bâtiment dans les systèmes de chauffage ou de ventilation par soufflage. Afin de conserver un niveau de confort 


\section{Nomenclature}

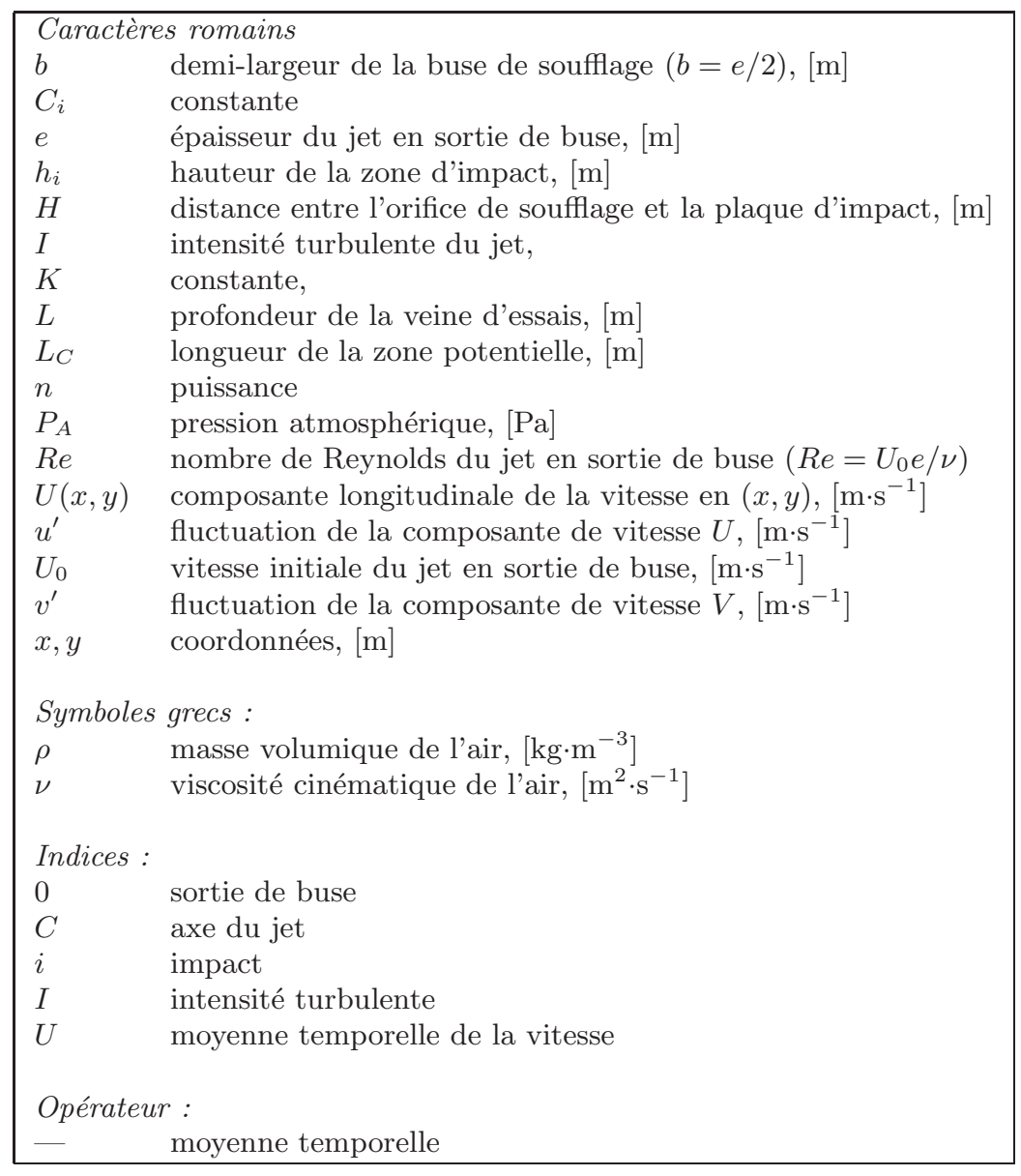

correct aux usagers tout en assurant un débit suffisant, il est ici important de connaître le champ de vitesse du jet. La bonne connaissance des lois de vitesse est également indispensable pour le développement de modèles zonaux capables de restituer de manière globale les transferts de masse dans le local [2].

Des études précédentes ont montré que l'optimisation de tous ces dispositifs passait par une meilleure compréhension de la cinématique du jet. Pour étudier et optimiser de telles installations, il est souvent nécessaire de passer par la réalisation de maquettes à échelle réduite car les installations réelles peuvent être de taille relativement importante.

Ainsi un modèle global décrivant la décroissance de la vitesse axiale, valable pour différentes valeurs du rapport $H / e$ et pour une gamme assez large du nombre de Reynolds, est intéressante à plusieurs égards. Il peut constituer un outil de validation de résultats numériques sans avoir recours systématiquement à l'expérimentation. Ce peut également être un outil de pré-dimensionnement d'installation, par exemple pour déterminer les zones d'écoulement où la vitesse de l'air reste comprise dans une certaine gamme. Enfin ce modèle de décroissance peut servir au développement de modèles zonaux globaux comme cités précédemment.
Même si la plupart des auteurs s'accordent sur les propriétés générales des jets libres (décroissance de la vitesse axiale, profils affines), nous notons une forte disparité des données bibliographiques par exemple sur la longueur du noyau potentiel et la loi d'épanouissement du jet. Cette diversité de résultats est probablement due à des différences de conditions aux limites des jets étudiés (géométrie des buses, profils de vitesse et d'intensité de turbulence à l'orifice). Dans ces conditions, l'exploitation des résultats de la littérature est très délicate. Pour cette raison, nous avons réalisé une soufflerie à géométrie variable, permettant de reproduire à échelle réduite $(1 / 10$ à environ $1 / 2)$ les installations industrielles réelles.

\section{Physique des jests d'air plans}

Bien que le jet plan ait été largement étudié dans le passé [3], il subsiste des zones d'ombre, particulièrement au niveau de la zone d'impact. De surcroît, très peu d'études traitent des effets de dimensions géométriques, tel que le rapport $H / e$.

En fonction du rapport géométrique $H / e$, le champ de vitesse d'un jet en impact montre deux, trois ou quatre régions très différentes du point de vue cinématique. 


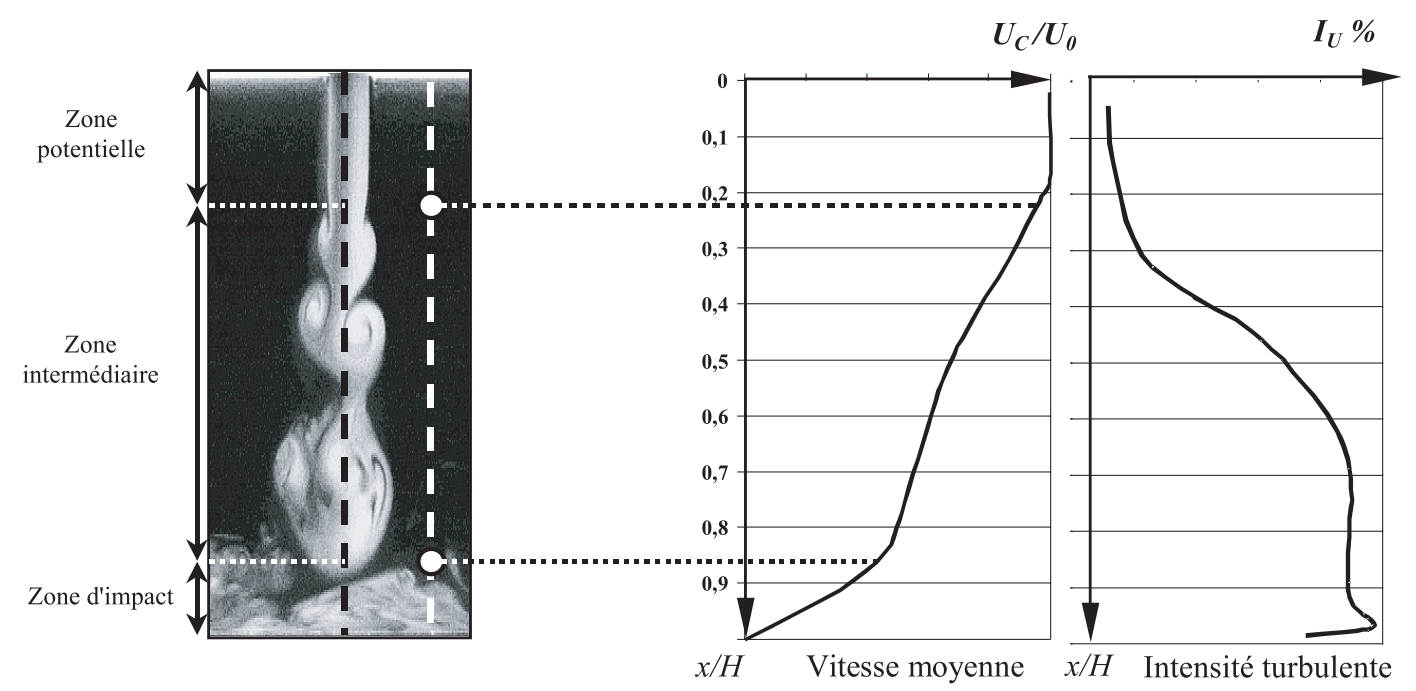

Fig. 1. Visualisation d'un jet en impact et distribution schématique de $U_{C} / U_{0}$ et $I_{U}$.

Pour les rapports $H / e$ importants, on peut distinguer comme pour le jet libre, la zone potentielle, la zone de transition, la zone d'affinité ou développée et en plus, la zone dite de recompression ou d'impact. Un schéma de principe de la distribution de vitesse et de l'intensité turbulente d'un tel jet est montré sur la figure 1 .

\subsection{Zone potentielle}

Dans cette zone, la vitesse axiale reste constante et égale à la vitesse en sortie de buse. La longueur de la zone potentielle varie entre $3 e$ et $6 e$ et dépend fortement des conditions initiales. De nombreux auteurs annoncent que la longueur de cette zone ne varie pas avec le nombre de Reynolds [4-7]. D'autres, au contraire, montrent qu'elle dépend du régime de l'écoulement, [8-10]. Par contre tous les auteurs constatent que cette longueur dépend de l'intensité de la turbulence initiale. Van et Howell [11] proposent une relation empirique entre la longueur de cette zone et l'intensité de la turbulence :

$$
L_{C}=5,39-0,266 I_{0}
$$

Sunyach et Mathieu [12] expliquent les fluctuations de la longueur du cône par une interaction entre les deux couches de mélange. Namer et Ötügen [8] indiquent que la formation des structures cohérentes dans les couches de cisaillement favorisent ces fluctuations de la longueur et de la largeur de la zone potentielle.

Thomas et Goldschmidt [13] complètent ces résultats par une analyse des fluctuations de vitesse dans les couches de mélange. Ils mettent en évidence plusieurs modes de fluctuations. Il semble exister une combinaison de fluctuations symétriques et anti-symétriques (modes sinueux et variqueux). D'après Garimella et Nenaydykh [14], et, Ashforth et Jambunathan [15], d'autres paramètres, tels que la forme de la buse de soufflage ou la distribution de vitesse en sortie de buse, conditionnent le phénomène.

\subsection{Zone de transition}

Dans cette zone, la solution analytique du champ de vitesse d'un jet libre présentant une faible intensité turbulente est donnée par l'équation (2) [16]. Notons que cette expression est applicable aussi bien pour la zone potentielle que pour la zone de transition :

$$
\frac{U(x, y)}{U_{0}}=\frac{1}{2}\left[1-\operatorname{erf}\left(C_{1} \frac{y-\frac{e}{2}}{x}\right)\right] \text { avec } \quad C_{1}=13,5
$$

\subsection{Zone développée ou d'affinité}

De nombreux auteurs ont essayé de définir une loi universelle permettant de décrire l'évolution de la vitesse axiale. Ces équations sont obtenues, pour la plupart, de manière empirique. Du fait des différences au niveau des conditions expérimentales, ces lois présentent une disparité importante, ce qui en limite le domaine d'utilisation. Malgré tout, dans le cas du jet plan libre, la plupart des auteurs s'accordent sur l'évolution de cette vitesse axiale qui peut s'écrire sous la forme suivante (Éq. (3)) :

$$
\frac{U_{c}(x)}{U_{0}}=C_{2}\left(\frac{x}{e}-C_{3}\right)^{-\frac{1}{2}}
$$

Les valeurs des constants $C_{2}$ et $C_{3}$ diffèrent notablement entre deux études. Par exemple, $C_{2}$ varie entre 1,9 et 3 tandis que $C_{3}$, également appelée origine fictive $\mathrm{du}$ jet, oscille entre -8 et +10 [17]. Pour un jet libre, Schlichting [16] montre que le champ de vitesse $U(x, y)$ et la vitesse sur l'axe peuvent être calculés à l'aide de :

$$
\frac{U(x, y)}{U_{c}(x)}=\frac{\sqrt{3}}{2} \sqrt{\frac{7,67 e}{x}}\left[1-\tanh ^{2}\left(7,67 \frac{y}{x}\right)\right]
$$

De plus, une grande disparité des résultats apparaît quant à la distance pour laquelle les profils de vitesse 


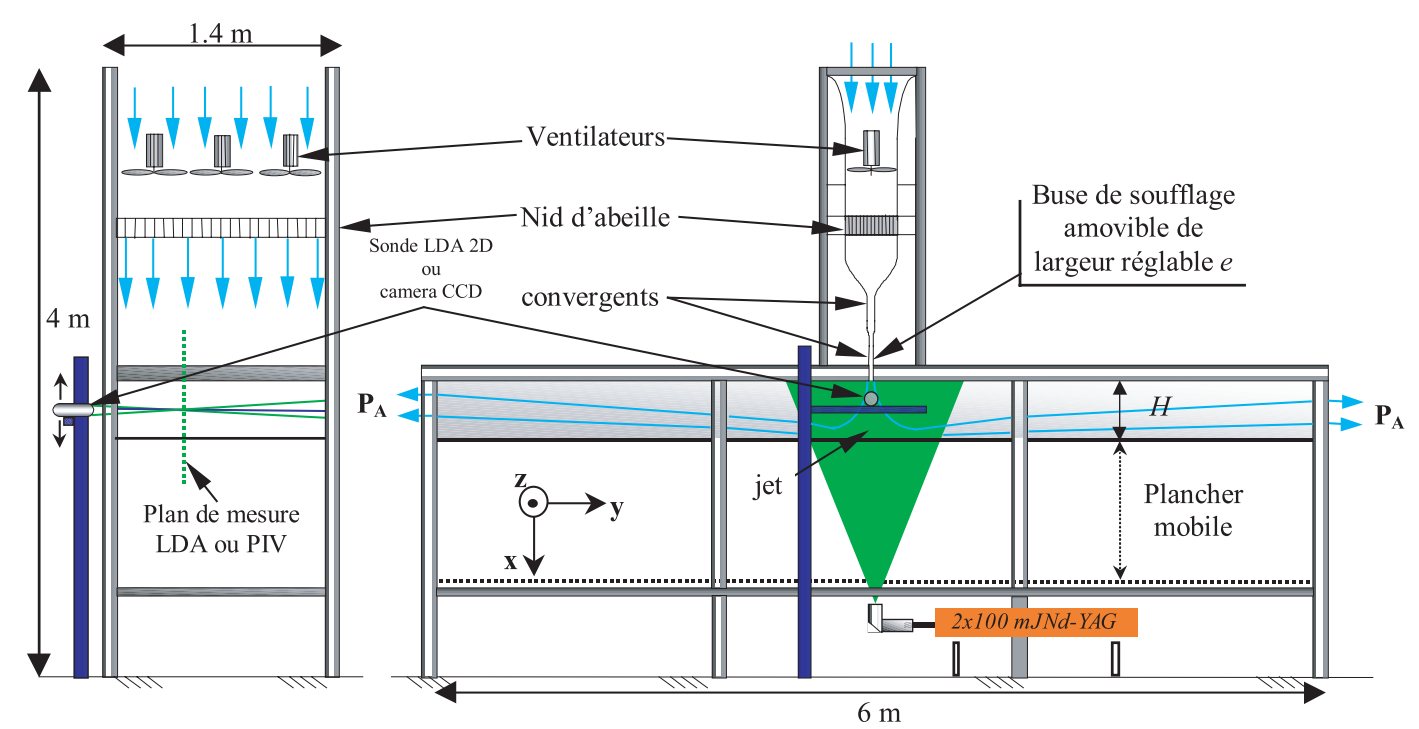

Fig. 2. Vue schématique du banc d'essai.

deviennent similaires : 20e pour Antonia et al. [6], 25e pour Jenkins et Goldschmidt [18], 40e pour Bashir et Uberoi [19].

Pour le jet en impact, Beltaos et Rajaratnam [4] insèrent un facteur additionnel qui fait intervenir le rapport $H / e$. La relation qu'ils obtiennent est valable en aval de la zone potentielle jusqu'à $x / H=0,65$, et s'écrit sous la forme suivante :

$$
\frac{U_{c}(x)}{U_{0}} \sqrt{\frac{H}{e}}=C_{4}\left(\frac{x}{H}\right)^{\frac{1}{2}} \quad \text { avec } \quad C_{4}=2,5
$$

Avec cette expression, il est possible de calculer la vitesse axiale quel que soit le rapport $H / e$. Le coefficient $C_{4}$, est obtenu empiriquement mais la grande majorité des auteurs trouve une valeur proche de 2,5 .

\subsection{Zone d'impact ou de recompression}

Dans le cas d'un jet en impact, une quatrième zone apparaît, elle se nomme habituellement zone de recompression ou zone d'impact. Selon Gutmark et al. [20], l'influence de cette zone s'étend approximativement sur $25 \%$ de la hauteur $H$, comptée à partir de l'impact.

Très peu d'auteurs se sont intéressés à cette zone du point de vue cinématique. Cependant, quelques uns d'entre eux présentent des résultats comme Tani et Komatsu [21] pour le jet axisymétrique, Mathieu [22], Sakakibara et al. [23] pour le jet plan. Beltaos et Rajaratnam [4] apportent des informations supplémentaires. En effet, ils suggèrent, une expression semi-analytique (Éq. (6)) décrivant la diminution de la vitesse sur l'axe, valable pour $0,75 \leq x / H \leq 0,98$ :

$$
\frac{U_{c}(x)}{U_{0}} \sqrt{\frac{H}{e}}=C_{5} \sqrt{1-\frac{x}{H}} \quad \text { avec } \quad C_{5}=5,5
$$

La valeur de $C_{5}$ obtenue expérimentalement est 5,5; malheureusement, elle dépend fortement des conditions opératoires. Les auteurs ont également affiné leur analyse en présentant une loi linéaire pour $x / H>0,98$. Dans cette zone, ils proposent la loi suivante :

$$
\frac{U_{c}(x)}{U_{0}} \sqrt{\frac{H}{e}} \cong C_{6} \frac{x}{H} \quad \text { avec } \quad C_{6}=30
$$

\section{Méthodologie expérimentale}

Ce banc, à géométrie variable, représenté sur la figure 2, permet de créer un jet plan de largeur de buse $e$ comprise entre $20 \mathrm{~mm}$ et $80 \mathrm{~mm}$ et de longueur (profondeur) $L$ égale à $1400 \mathrm{~mm}$. Les mesures de vitesse ont été réalisées sur des jets plans turbulents de différentes tailles.

Le système de soufflage permet d'atteindre des nombres de Reynolds valant 27000 . L'impact est réalisé par un plancher placé orthogonalement au jet à une distance $H$ ajustable et comprise entre 100 et $1200 \mathrm{~mm}$. Il est ainsi possible de conserver un rapport $H / e$ fixe ou de le faire varier entre 5 et 50 . Une analyse paramétrique a été conduite afin d'observer l'influence de grandeurs caractéristiques sur le développement cinématique du jet. Les principaux paramètres retenus pour l'étude sont le nombre de Reynolds $R e$ et le rapport $H / e$. Les campagnes de mesure permettent d'accéder aux champs de vitesse par vélocimétrie laser et par imagerie de particules (PIV).

Les vitesses moyennes et les grandeurs turbulentes sont mesurées à l'aide d'un anémomètre laser à effet Doppler à deux composantes. La tête optique est fixée sur un système de déplacement micrométrique; sa position est connue avec une précision de $10 \mu \mathrm{m}$. La focale du système optique est de $160 \mathrm{~mm}$, ce qui conduit à un volume de mesure d'approximativement $75 \times 75 \times 635 \mu \mathrm{m}^{3}$. Le système est piloté par un $\mathrm{PC}$ et les données sont traitées par un FVA (Flow Velocity Analyser) distribué par la société DANTEC ${ }^{\circledR}$. Les particules de traçage sont de fines gouttelettes de glycol produites par un générateur de brouillard de marque MARTIN. Le diamètre moyen 


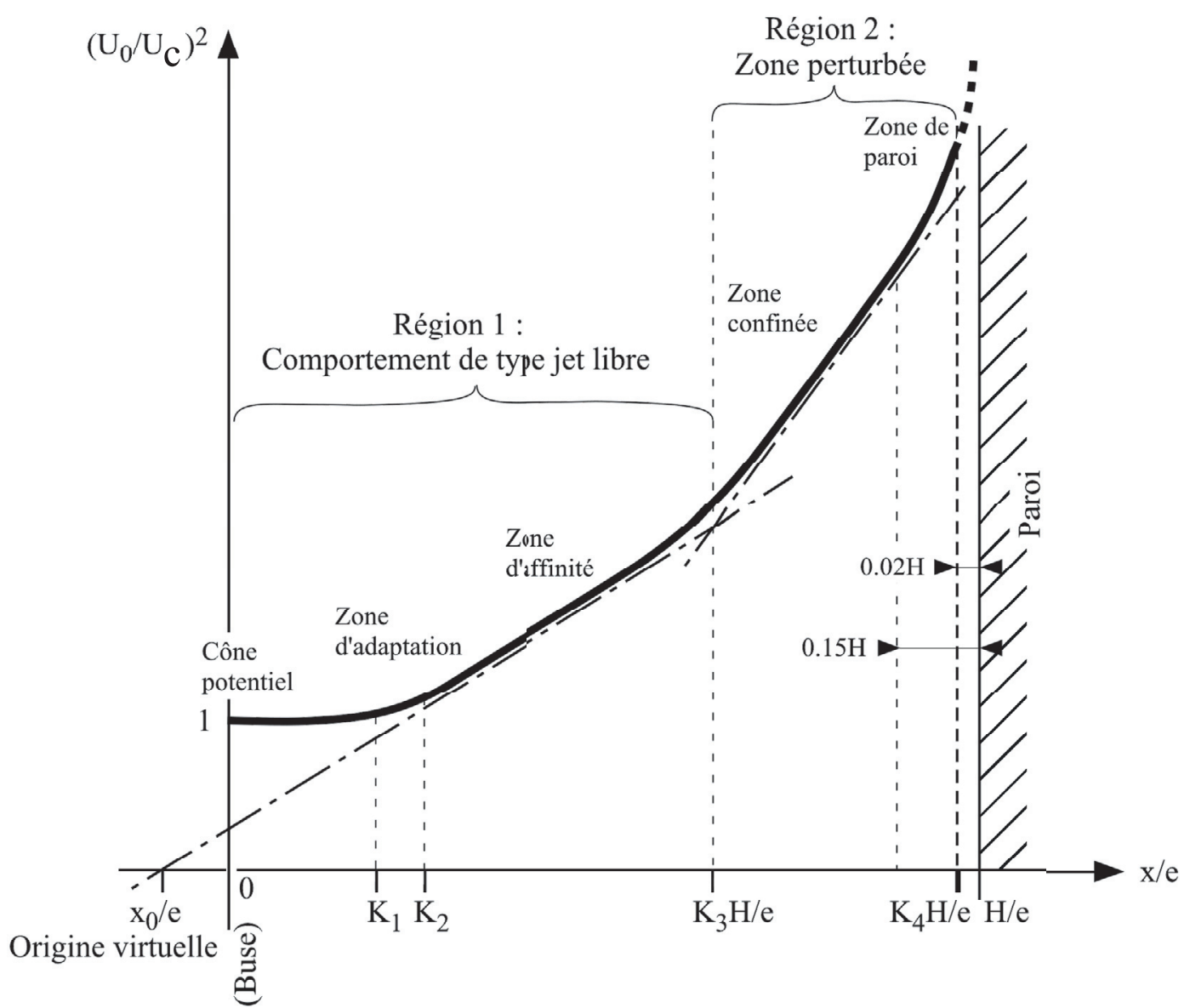

Fig. 3. Schéma de principe du découpage par zones caractéristiques du jet.

des particules, mesuré par un granulomètre laser, vaut $3 \mu \mathrm{m}$. L'ensemencement est réalisé, d'une part, juste en aval des ventilateurs de soufflage et d'autre part de chaque côté du jet afin de limiter les biais de mesures conditionnelles. 50000 mesures sont collectées pour chaque point sondé, la fréquence d'acquisition est de l'ordre de $1 \mathrm{kHz}$. L'échantillonnage est réalisé pendant une minute. L'incertitude globale est estimée à $\pm 2,5 \%$ de la vitesse moyenne dans le pire des cas.

\section{Modélisation semi-analytique de la décroissance de la vitesse axiale}

Nous décrivons ici, la méthode d'élaboration d'un modèle global et semi-empirique capable de décrire l'évolution de la vitesse moyenne dans le plan de symétrie d'un jet plan en impact, quelles que soient les conditions de confinement. Ce modèle est compatible avec la physique de ce type d'écoulement lorsqu'on s'appuie sur le découpage en zones explicité dans ce qui suit (Fig. 3). Notons que le modèle n'est applicable que pour des rapports $H / e>5$.

L'écoulement caractéristique d'une situation de type « jet plan en impact sur une plaque plane normale au flux principal » peut grossièrement être divisé en deux régions :
- une région initiale où l'écoulement demeure insensible à la présence de la plaque (si celle-ci est suffisamment éloignée de la buse de soufflage). L'écoulement est alors conforme à celui d'une situation de type « jet libre débouchant dans un milieu au repos »;

- une région dite perturbée au sein de laquelle les propriétés d'entraînement de l'écoulement sont modifiées par rapport à une situation de type jet libre.

Dans la première région de l'écoulement, les raisonnements ont été conduits en considérant la distance à la buse soit $x / e$. Dans la seconde partie de l'écoulement, les effets de confinement dominent. Le modèle a été construit en raisonnant sur la distance à l'impact, c'est-à-dire $x / H$ plutôt que $x / e$ qui a alors moins de signification.

\subsection{Région initiale de l'écoulement}

On y distingue 3 zones : le cône à potentiel, une zone développée et, entre les deux, une zone de raccordement (ou d'adaptation) assurant la continuité de l'évolution des grandeurs caractéristiques du jet sur le plan de symétrie.

Dans cette région, on considère que le jet n'est pas perturbé par l'impact. La présence de la plaque en aval de la buse de soufflage n'a d'influence ni sur les mécanismes d'entraînement ni sur la quantité d'air mis en mouvement 


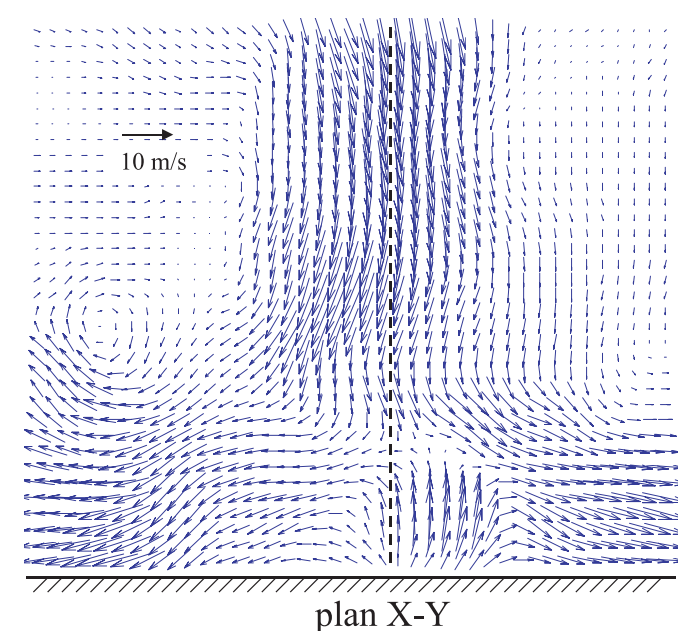

plan X-Y

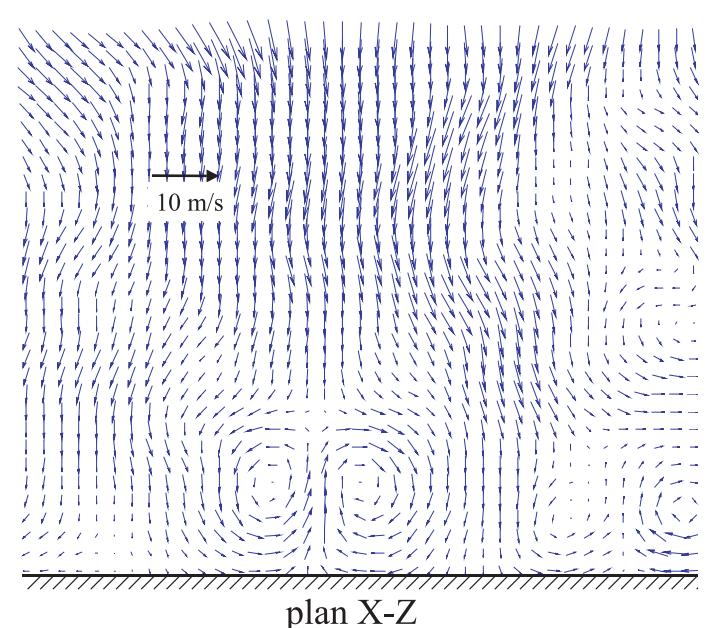

plan X-Z

Fig. 4. Champ des vecteurs vitesse instantanée dans la zone d'impact $R e=2,7 \times 10^{4}-e=20 \mathrm{~mm}$.

par le jet à ce niveau de l'écoulement. La loi d'évolution de la vitesse moyenne dans le plan de symétrie du jet se déduit de la théorie du jet libre établie en raisonnant sur la conservation de la quantité de mouvement et l'accroissement du débit masse par entraînement non confiné. Sans tenir compte pour l'instant de l'origine fictive, la relation proposée doit donc être cohérente avec les résultats connus suivants :

- $U_{C} / U_{0}=1$ pour $x / e<5$;

- $U_{C} / U_{0}=B(x / e)^{-0,5}$ dans la zone développée (voir Fig. 3).

Cette dernière description n'est valable que si les conditions d'affinité des profils transversaux de la vitesse moyenne et des tensions de Reynolds sont vérifiées. Cette condition a été vérifiée expérimentalement. Puisque l'écoulement se comporte comme un jet libre, il n'y a aucune raison de modifier la valeur de $B$. On garde par conséquent $B=2,454$. Notons que pour le jet libre, cette relation est valable pour $x / e>10$ où $e$ est la largeur de buse. La zone développée peut apparaître plus tôt dans un jet en impact selon la valeur du rapport $H / e$.

Le raccordement entre le cône à potentiel et la zone développée est assuré par l'introduction de fonctions en puissance $n$ de la variable sans dimension $x / e$. Ces fonctions permettent également de rendre compte mathématiquement de la contraction de la zone développée, voire sa disparition, lorsque le rapport $H / e$ est inférieur à 20.

\subsection{Région perturbée}

Dans cette région, l'écoulement est influencé par la surface d'impact. La déflection du jet à l'impact conduit simultanément à une décélération verticale de l'écoulement et à une accélération progressive dans les directions transversales. On y distingue 2 zones : une zone dite de confinement et une zone de paroi (Fig. 3).

Dans la première zone, la quantité de mouvement est naturellement conservée. Toutefois, le confinement causé par la présence d'une surface d'impact a pour effet de réduire l'alimentation externe en air du jet par rapport à une situation de jet libre. Simultanément, le taux d'épanouissement du jet augmente par rapport au cas du jet libre. Les fonctions en puissance $n$ introduites dans le modèle permettent de moduler le terme qui, dans la loi classique de décroissance de la vitesse moyenne dans la zone d'affinité du jet libre, est représentatif du débit d'air entraîné $^{1}$ par le jet, soit le coefficient $B$.

La zone de paroi est un domaine de transition entre la zone de confinement précédente et la région de l'écoulement, juste avant l'impact, où le jet initial se sépare en deux jets de paroi de direction opposée.

Nous continuons de formuler l'hypothèse selon laquelle la quantité de mouvement est conservée. Les effets visqueux dus à la déviation de l'écoulement et à la paroi sont négligés. En revanche, dans cette zone nous interdisons volontairement tout accroissement de la masse d'air ambiant mis en mouvement par le jet. Le mécanisme retenu est alors un simple effet de déviation du jet par le plan d'impact perpendiculaire.

Dans le plan de symétrie du jet, la composante longitudinale de la vitesse devrait s'annuler à la paroi. Or, les expériences montrent, en amont de la paroi, l'existence d'une ligne d'arrêt avant déviation complète de l'écoulement moyen. La loi de décroissance de la vitesse moyenne dans le plan de symétrie du jet rend compte de ces observations.

Toutefois, le modèle conduit à des valeurs négatives de la vitesse dans la zone de paroi. Ce résultat, vrai instantanément en raison de la présence de tourbillons de type Goërtler (Fig. 4) n'est pas réaliste en moyenne. La loi de décroissance est donc arbitrairement mise à 0 pour $x / H>K_{4}\left(\right.$ ou $\left.x / e>K_{4}\right)$.

La formulation générale de la loi est la suivante, les différents coefficients ont été obtenus par « calage »avec les données expérimentales. La validation est présentée

\footnotetext{
1 Rappel : à l'abscisse $x$, le débit d'entraînement est donné via $Q_{\text {total }} / Q_{0}=3\left[\left(x-x_{0}\right) / e\right]^{0,5} / 2 B$.
} 


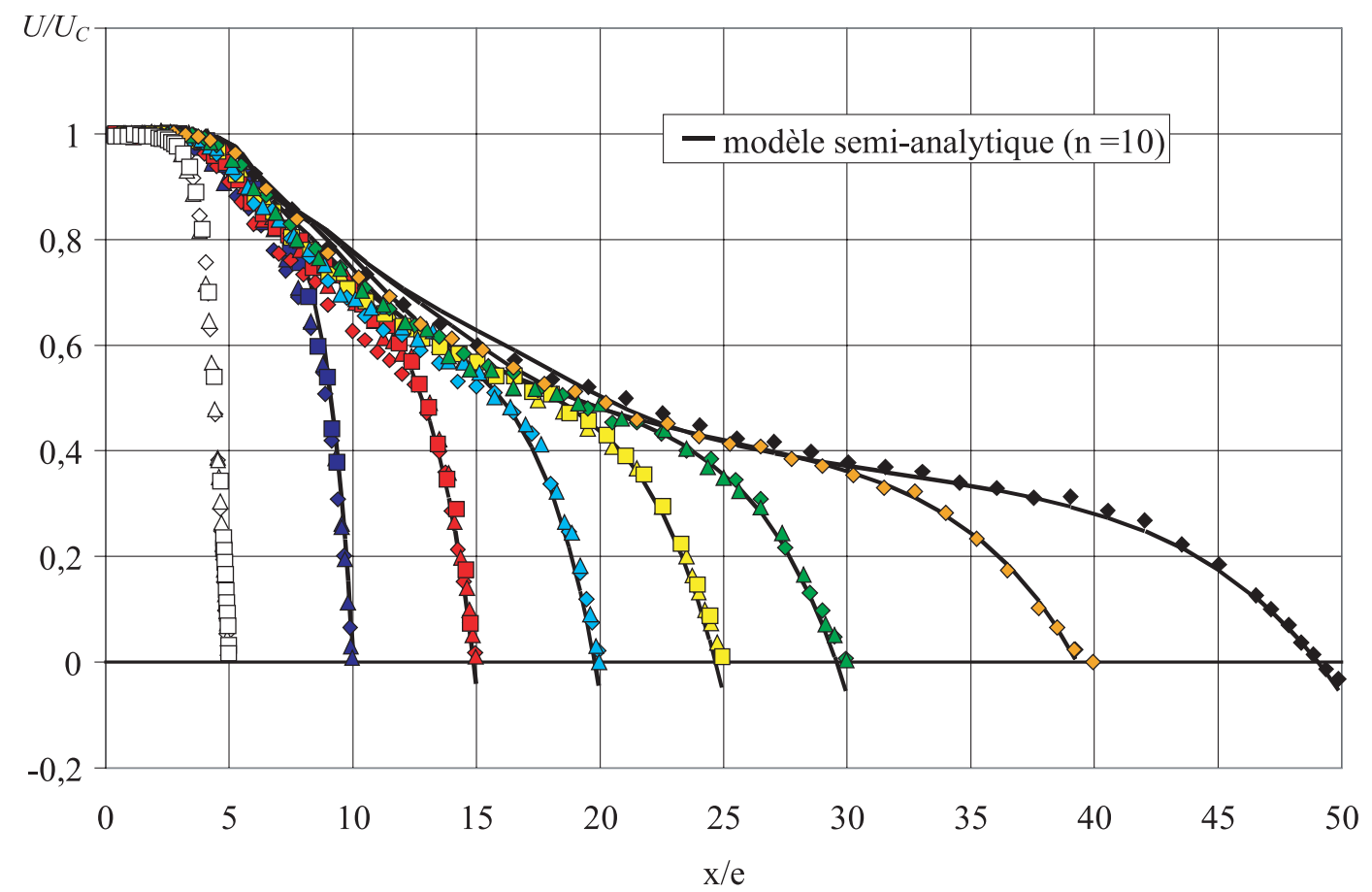

Fig. 5. Décroissance de la vitesse axiale en fonction de $x / e$ pour $5<H / e<50 . \diamond: e=20 \mathrm{~mm} ; \circ: e=40 \mathrm{~mm} ; \square: e=8 \mathrm{~mm}$.

dans le paragraphe 5

$$
\begin{aligned}
\frac{U_{c}}{U_{0}}= & {\left[\frac{\left(\frac{H}{e}\right)^{n}+\frac{A}{2,454}\left(\frac{x}{K_{3} e}\right)^{n}}{\left.\left(\frac{H}{e}\right)^{n}+\left(\frac{x}{K_{3} e}\right)^{n}\right]}\right.} \\
& {\left[1+2,454\left(\frac{x}{K_{2} e}\right)^{n}\left(1-\frac{1}{1+\left(\frac{x}{K_{1} e}\right)^{n}}\right)\left(\frac{x}{e}\right)^{-\frac{1}{2}}\right.} \\
& \left.\left(1-\left(\frac{H}{e}\right)^{-n}\left(\frac{x}{K_{4} e}\right)^{n}\right)\right] \\
\times & \times\left[\frac{1}{1+\left(\frac{x}{K_{2} e}\right)^{n}}\right]
\end{aligned}
$$

Les valeurs des constantes empiriques sont les suivantes :

$$
\begin{aligned}
n & =10 \\
K_{1} & =6,5 \\
K_{2} & =7,5 \\
K_{3} & =0,4 \\
K_{4} & =0,98 \text { ou } \quad K_{4}=\frac{1}{1+0,02 \operatorname{arctg}\left(0,03 \frac{H}{e}\right)}
\end{aligned}
$$

$$
\begin{aligned}
& A=2,045 \text { ou } \\
& A=2,045+0,409\left[1-\exp \left(-0,0008\left(\frac{H}{e}-50\right)^{2}\right)\right]
\end{aligned}
$$

Dans le modèle, la prise en compte de l'origine fictive $x_{0}$ du jet permet d'intégrer globalement les effets des conditions initiales. En effet, pour un nombre de Reynolds $(R e>8000)$ et pour un rapport $(H / e>10)$ donnés, les résultats expérimentaux présentés par la suite révèlent parfois une légère dispersion. La dispersion pourrait être plus large si l'on ajoutait les résultats de la littérature. Ces écarts, on le sait, sont souvent dus à des différences au niveau des conditions initiales. En particulier, la conservation du rapport $H / e$ pour différentes valeurs de $e$ ne garantit pas la similitude des épaisseurs de couche limite en sortie de buse.

\section{Résultats expérimentaux}

\subsection{Distribution de vitesse axiale}

La figure 5 présente l'évolution axiale de la vitesse moyenne de l'écoulement (composante suivant $x$ ), obtenue par LDV pour des rapports compris entre 5 et 50 . On observe une forte influence de ce rapport sur le développement de jet, excepté dans la région du cône potentiel quel que soit $H / e$. Par ailleurs, un regroupement des zones de transition et développée apparaît quand ce rapport est inférieur à 15. Enfin, quand on conserve constant le nombre de Reynolds et le rapport $H / e$ tout en faisant varier $e$ de 20 à $80 \mathrm{~mm}$, on constate que le développement des grandeurs cinématiques moyennes du jet à nombre de Reynolds constant est identique pour les trois cas. En conséquence, il a été fixé à $R e=2,7 \times 10^{4}$. 


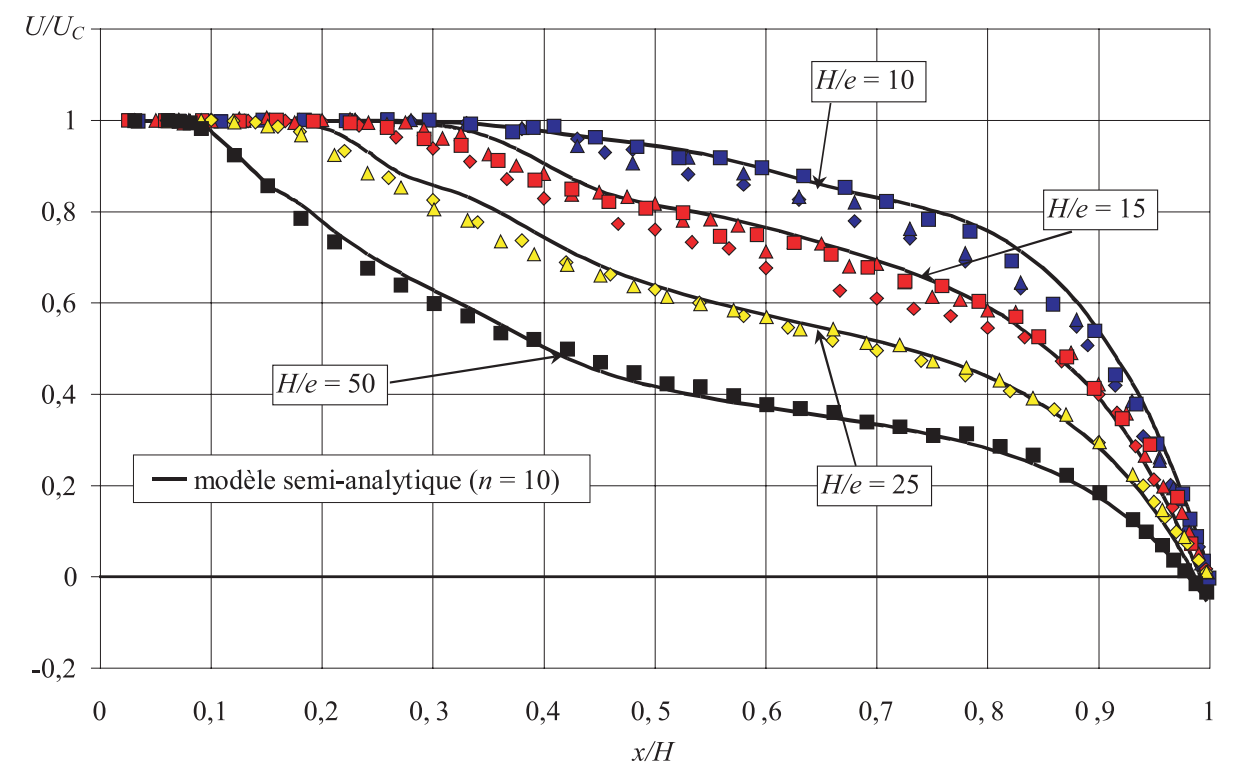

Fig. 6. Comparaison expérience/modèle pour différents cas de jet. $\diamond: e=20 \mathrm{~mm} ; \circ: e=40 \mathrm{~mm} ; \square: e=80 \mathrm{~mm}$.

\subsection{Validation du modèle de décroissance de la vitesse axiale}

Une fois le modèle défini, la deuxième étape consiste à comparer ce modèle avec les mesures expérimentales réalisées par vélocimétrie Doppler laser. Cette étape permettra d'ajuster le modèle en modifiant les coefficients empiriques $K_{1}, K_{2}, K_{3}, A$ et $B$ définis précédemment.

Basées sur une approche empirique, les valeurs retenues dans un premier temps sont : $K_{1}=6,5, K_{2}=7,5$; $\left(<K_{1}\right) ; K_{3}=0,4 ; A$ et $B$ étant fonctions de $H / e$ ou fixés respectivement à 2,045 et 1,02 . Avec ces valeurs, les deux modèles présentent cependant une zone mal représentée au niveau du raccordement entre le cône potentiel et la zone de décroissance en puissance $-1 / 2$. Même si les écarts sont tout à fait acceptables pour $H / e=50$ (inférieur à $4 \%$ ), ils deviennent trop importants pour $10<H / e<25$, et particulièrement pour $\mathrm{H} / \mathrm{e}=15$ (proche de $20 \%$ ). Outre les écarts relatifs importants, c'est surtout l'allure générale du modèle qui est non représentative de celle de la vitesse axiale.

C'est pourquoi, dans un deuxième temps, en conservant les valeurs des coefficients $K_{1}, K_{2}$ et $K_{3}$, la valeur de la puissance représentant la fonction de raccordement a été ajustée par essai/erreur. Nous avons conservé les caractéristiques du modèle à $A$ et $B$ variables, qui semblent être les mieux adaptées. Nous avons ensuite décrémenté le coefficient $n$ pour obtenir l'optimum à $n=10$. Pour ces valeurs des coefficients, la figure 6 compare les résultats obtenus à l'aide du modèle avec l'ensemble des données expérimentales.

\subsection{Demi-largeur du jet}

La demi largeur du jet, distance par rapport à l'axe pour laquelle la vitesse est moitié de celle sur l'axe, est obtenue à partir des profils transversaux de la composante longitudinale de la vitesse moyenne, mesurée à différentes distances de la buse de soufflage pour chaque largeur de buse. Quelques exemples de ces profils sont fournis sur les figures 7 à 10, dans le cas de la buse de $20 \mathrm{~mm}$ pour $H / e$ variant de 5 à 50 .

La figure 11 présente l'évolution de la demi largeur en fonction du rapport $H / e$, e variant de 20 à $80 \mathrm{~mm}$.

Ces figures mettent en évidence une forte corrélation entre le rapport $H / e$ et l'évolution de la demi-largeur du jet pour une même largeur de buse. Plus $H / e$ augmente, plus la demi-largeur croît. Ce phénomène est très visible dans la zone développée. Ce résultat est vérifié pour les trois largeurs de buse.

À l'aide de ces résultats, on peut représenter l'évolution de la demi-largeur en fonction du rapport $H / e$ dans la zone développée, c'est-à-dire à $x / H=0,6$. On comparera les résultats pour les trois largeurs de buse.

La figure 12 présente l'ensemble des résultats. Même si on observe une croissance de la demi-largeur quand $H / e$ augmente et quelle que soit la largeur de la buse, il est difficile de définir quantitativement cette évolution. On peut cependant constater que la croissance est quasiment linéaire.

\subsection{Distribution de l'intensité de la turbulence sur l'axe du jet}

Les figures 13 à 15 représentent l'évolution de l'intensité de turbulence de la composante $U$ en fonction du rapport pour une largeur de buse de 20, 40 et $80 \mathrm{~mm}$.

On constate que les profils deviennent similaires audelà d'un rapport de 20. Au-delà, l'évolution de l'intensité de la turbulence est quasiment la même quel que soit $H / e$, exceptée à l'approche de l'impact où l'on retrouve un pic de turbulence (représentatif de la zone d'impact) 


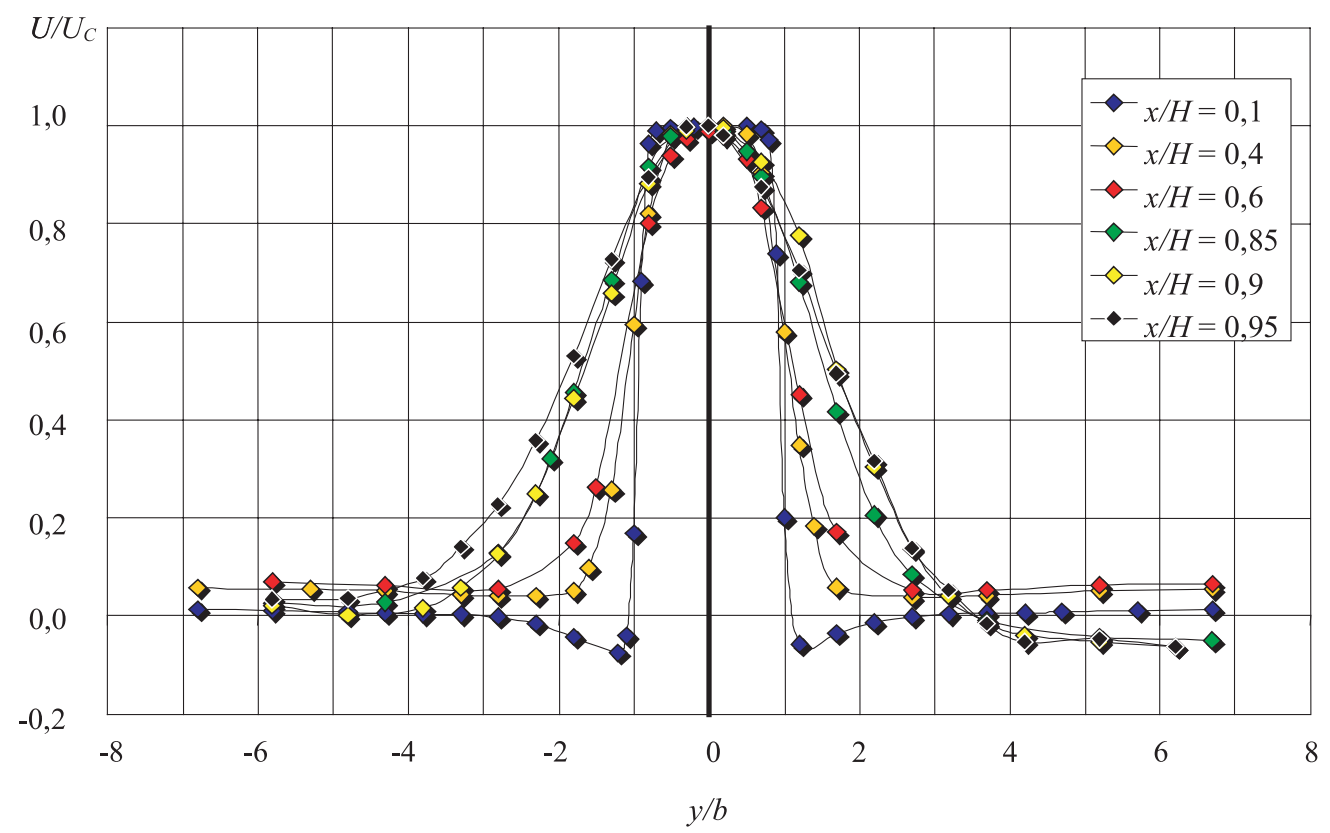

Fig. 7. Profils transversaux de la composante $U$ de la vitesse moyenne $e=20 \mathrm{~mm}, H / e=5, R e=27000$.

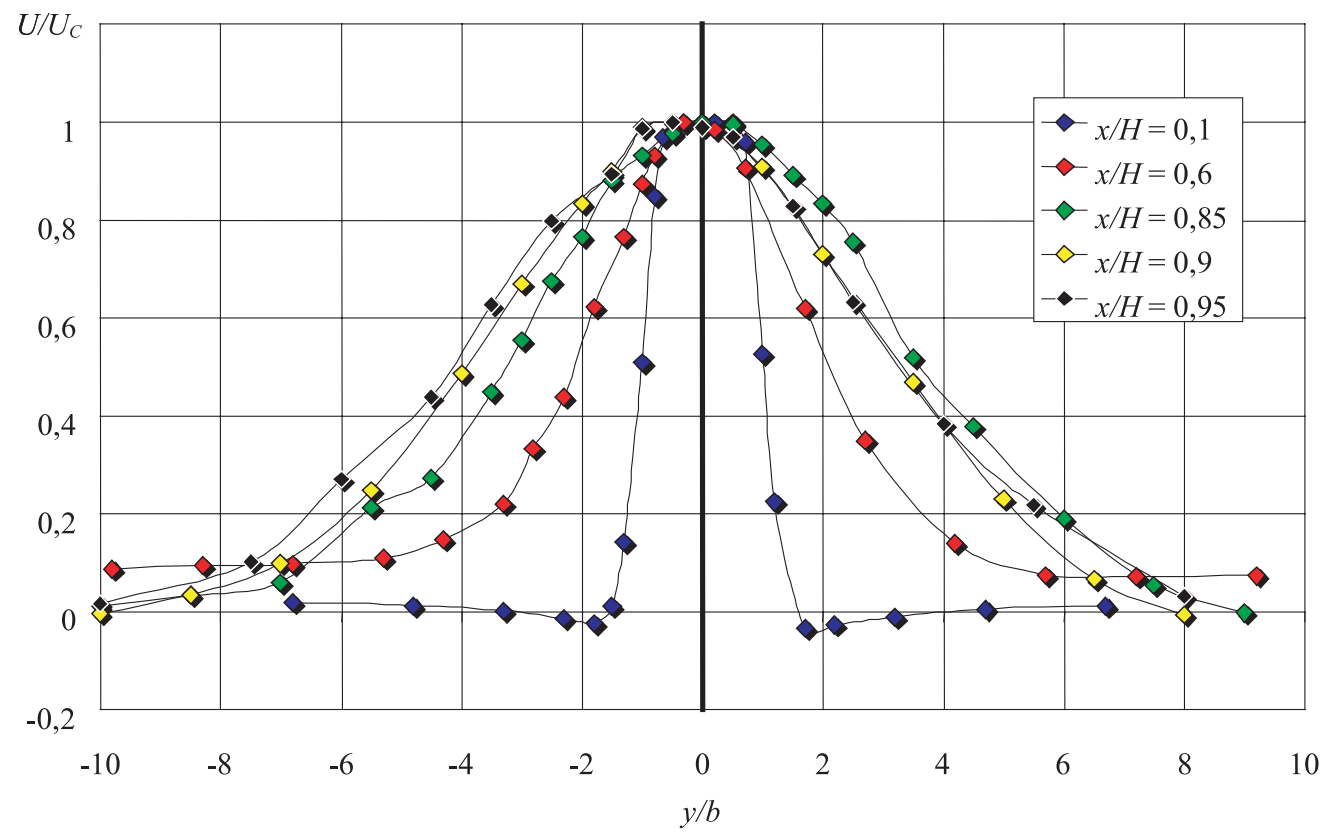

Fig. 8. Profils transversaux de la composante $U$ de la vitesse moyenne $e=20 \mathrm{~mm}, H / e=15, R e=27000$.

qui s'élargit quand $H / e$ augmente. Quand $H / e<20$, on observe un accroissement rapide de l'intensité de la turbulence que ce soit au niveau de la zone d'impact comme au niveau du maximum local situé dans la zone d'impact. Pour $H / e=5$, le jet change radicalement de comportement et l'intensité de la turbulence chute à des valeurs de l'ordre de $13 \%$ alors qu'elle atteignait près de $23 \%$ pour $H / e=10$. Ceci montre que la turbulence du jet est fortement accrue lorsque le rapport est compris entre 10 et 20 pour la buse de $20 \mathrm{~mm}$.
D'autre part, lorsque $H / e>20$, on remarque la présence d'un maximum local situé à une distance équivalente à environ $10 e$, quel que soit $H / e$. Notons qu'un maximum local est aussi observé pour $10 \leq H / e \leq$ 20. Ce maximum local précède une lente décroissance de pente constante pour tout $H / e$. La courbe passe ensuite par un minimum local avant la zone d'impact caractérisée par le pic de turbulence. Comparativement aux profils de vitesse moyenne, on peut considérer que cette évolution est représentative d'un jet développé. 


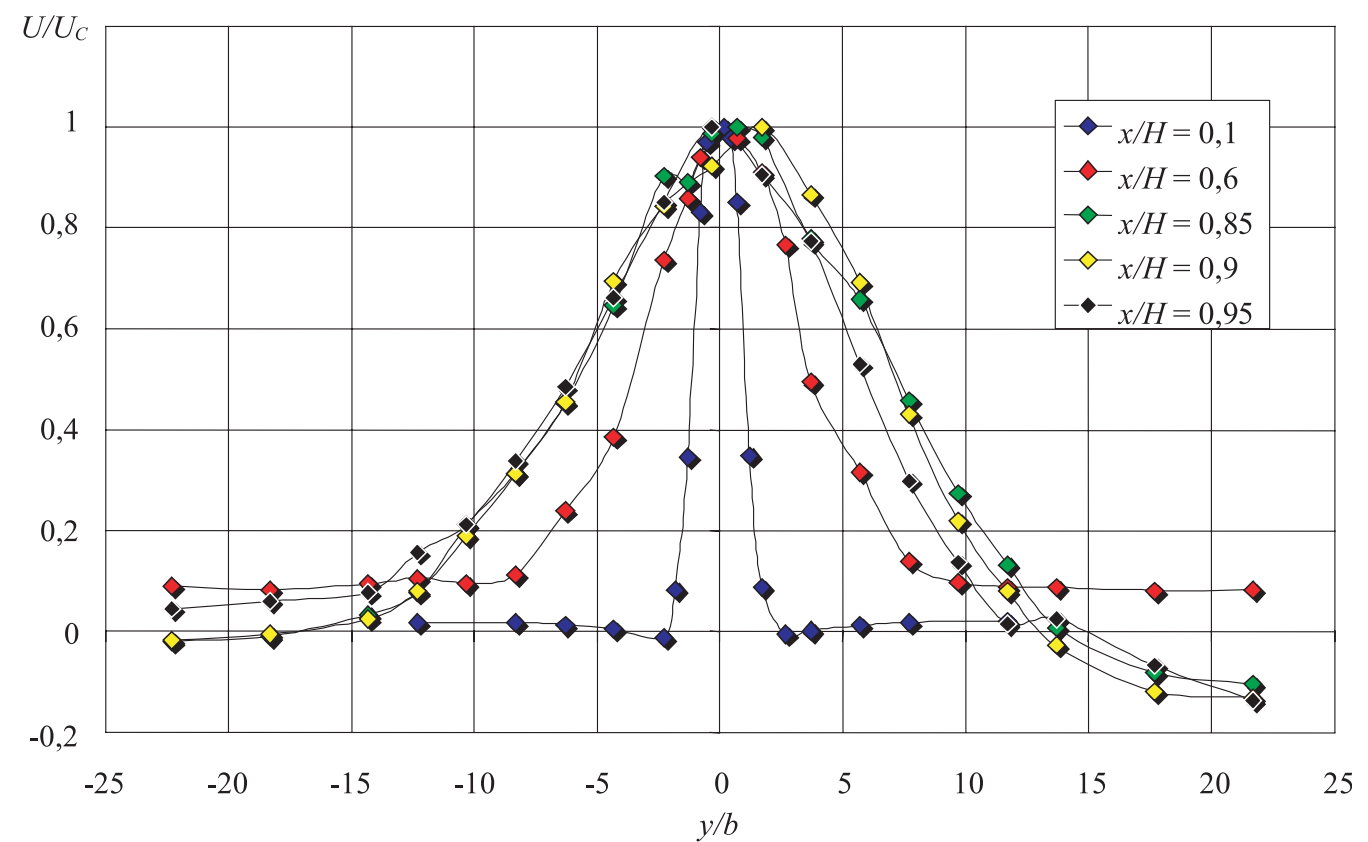

Fig. 9. Profils transversaux de la composante $U$ de la vitesse moyenne $e=20 \mathrm{~mm}, \mathrm{H} / e=25, R e=27000$.

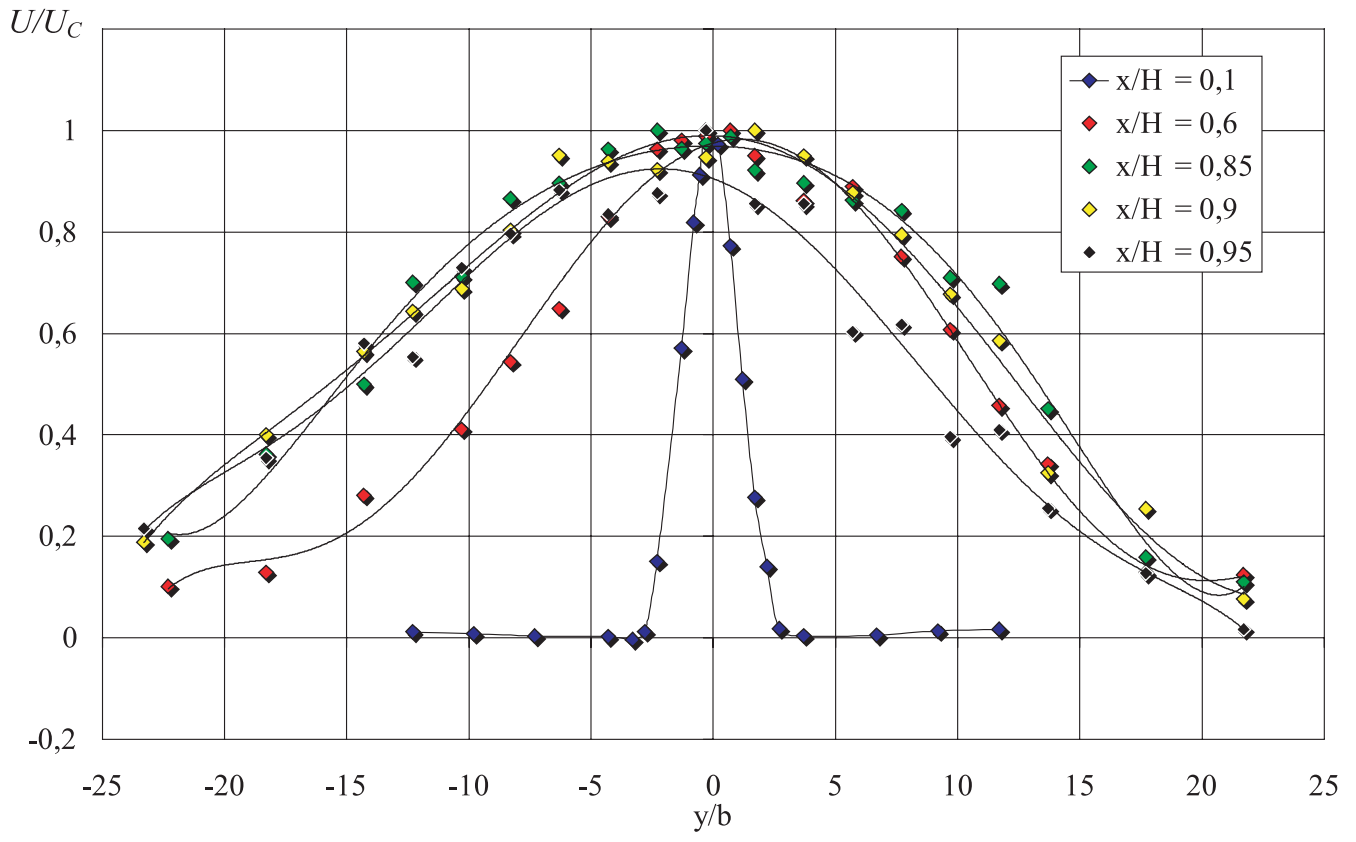

Fig. 10. Profils transversaux de la composante $U$ de la vitesse moyenne $e=20 \mathrm{~mm}, H / e=50, R e=27000$.

À partir des mesures obtenues pour $e=40 \mathrm{~mm}$, le maximum local se situe à environ $H / e=10$. La présence de ce maximum local pourrait être liée à la fin de la zone de transition lorsque les structures turbulentes sont complètement développées. En se référant à la littérature, il est assez difficile de définir précisément où s'achève la zone de transition et où commence la zone développée lorsqu'elle existe. La plupart des auteurs considèrent qu'un jet est pleinement développé à $20 e$ mais certains comme Schlichting [16] appliquent les lois de développement d'un jet développé à partir de $5 e$.
En revanche, la présence de ce maximum d'intensité de turbulence est bien mise en évidence. Même si la valeur de l'intensité de la turbulence diminue encore légèrement quand $H / e$ augmente, on peut estimer que la valeur de l'intensité de la turbulence tend vers $17 \sim 18 \%$ au fur et à mesure que $H / e$ croît.

En outre, on peut tracer en fonction de $H$ / $e$ l'évolution du pic d'intensité de la turbulence situé dans la zone d'impact. La figure 16 présente cette évolution pour les trois largeurs de buse. On remarque la présence d'un maximum 


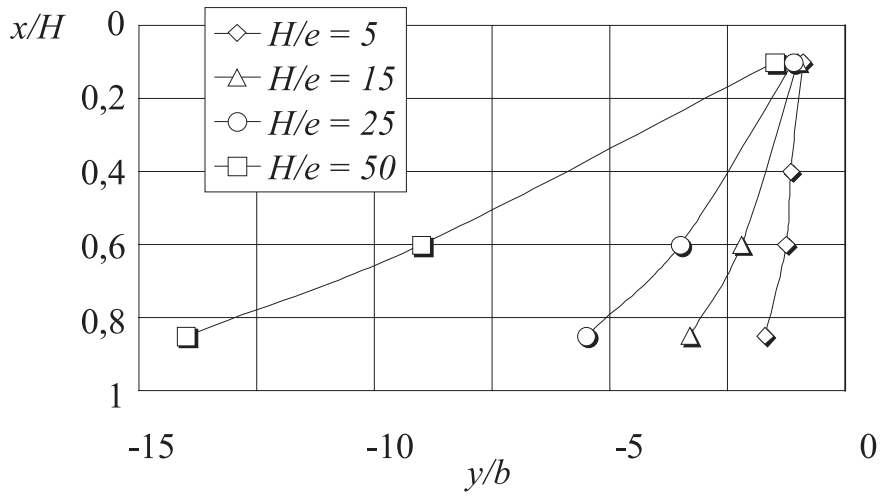

(a)

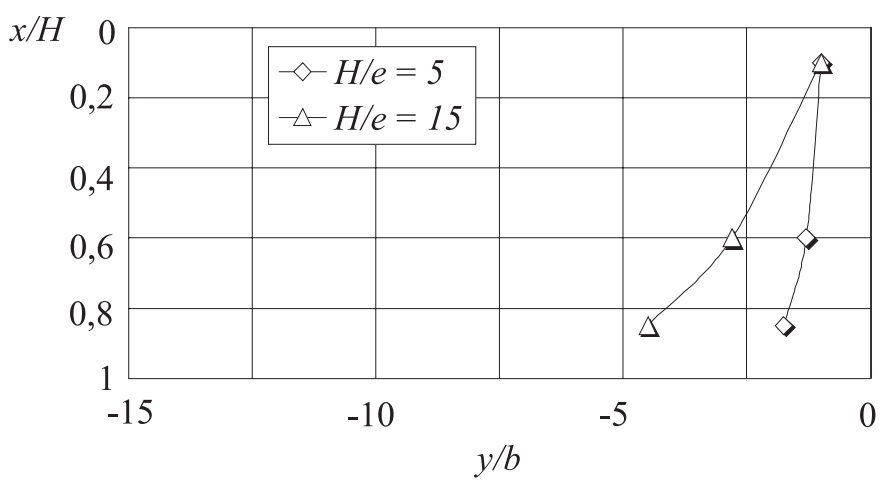

(c)

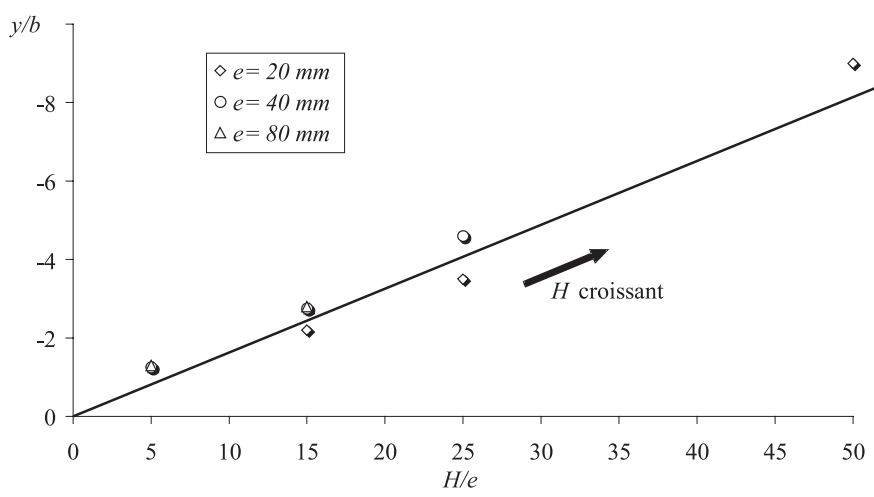

Fig. 12. Influence du rapport $H / e$ sur la demi-largeur pour $x / H=0,6$ et pour les trois largeurs de buse $(20,40$ et $80 \mathrm{~mm})$, $R e=27000$

à $H / e=10$, suivi d'une décroissance quasiment linéaire quand le rapport augmente.

\section{Conclusion}

Les premières investigations menées au cours de cette étude, ont consisté à quantifier le développement cinématique du jet. Les premiers résultats montrent que ce développement est indépendant du nombre de Reynolds dans la gamme de vitesses étudiée pour un rapport $H / e$ et une largeur de buse $e$ fixés.

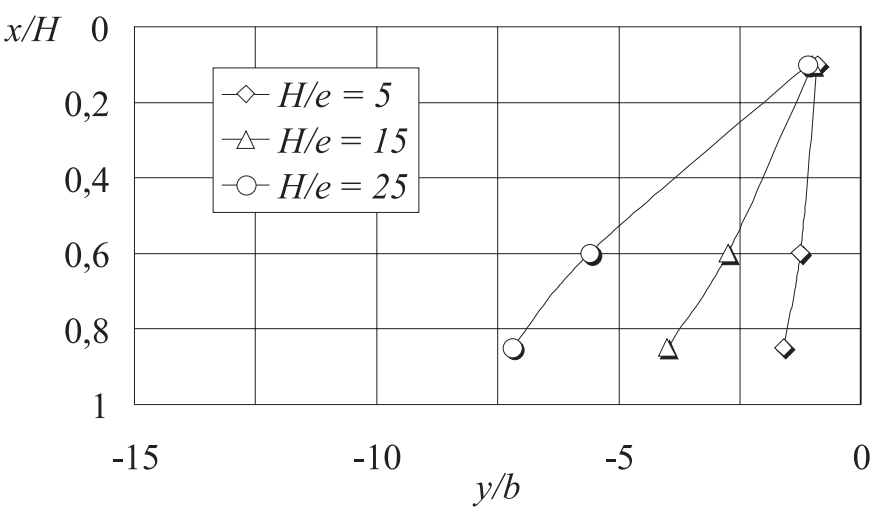

(b)

Fig. 11. Influence du rapport $H / e$ sur la demi-largeur du jet. (a) $: e=20 \mathrm{~mm}$; (b) $: e=40 \mathrm{~mm}$; (c) $: e=80 \mathrm{~mm}$; $R e=27000$.

Quand on fait varier le rapport $H / e$ entre 5 et 50 , on constate qu'il a une forte influence sur le développement des grandeurs cinématiques sur l'axe du jet. On observe par exemple un regroupement des zones de transition et développée quand ce rapport est inférieur à 15. Enfin, quand on fait varier les grandeurs géométriques de manière homothétique, tout en respectant la similitude de Reynolds, on constate que ce paramètre n'a aucune influence sur les lois d'évolution des grandeurs cinématiques moyennes du jet.

Les résultats présentés ici montrent bien la complexité du modèle à mettre en ouvre pour restituer correctement la décroissance de la vitesse axiale moyenne d'un jet plan turbulent en impact. Ceci est notamment dû au confinement produit par l'impact empêchant le développement latéral du jet et à l'apparition de tourbillons de TaylorGörtler dans la zone d'impact. Bien qu'un peu complexe, la loi semi-empirique proposée permet de restituer la décroissance de la vitesse axiale du jet dans une large gamme du rapport $H / e$. La prise en compte de la totalité des mécanismes supposés prédominants par notre modèle en fait un outil précieux, pour le développement de simulation à échelle réduite en laboratoire, ou pour la comparaison d'efficacité entre deux systèmes.

Les mesures de l'intensité de la turbulence sur l'axe du jet permettent de mettre en évidence l'apparition d'un extremum en deçà de $H / e=10$. Ce résultat laisse supposer que les transferts de masse ou de chaleur seront maximums entre $H / e=5$ et $H / e=10$. Ainsi, selon 


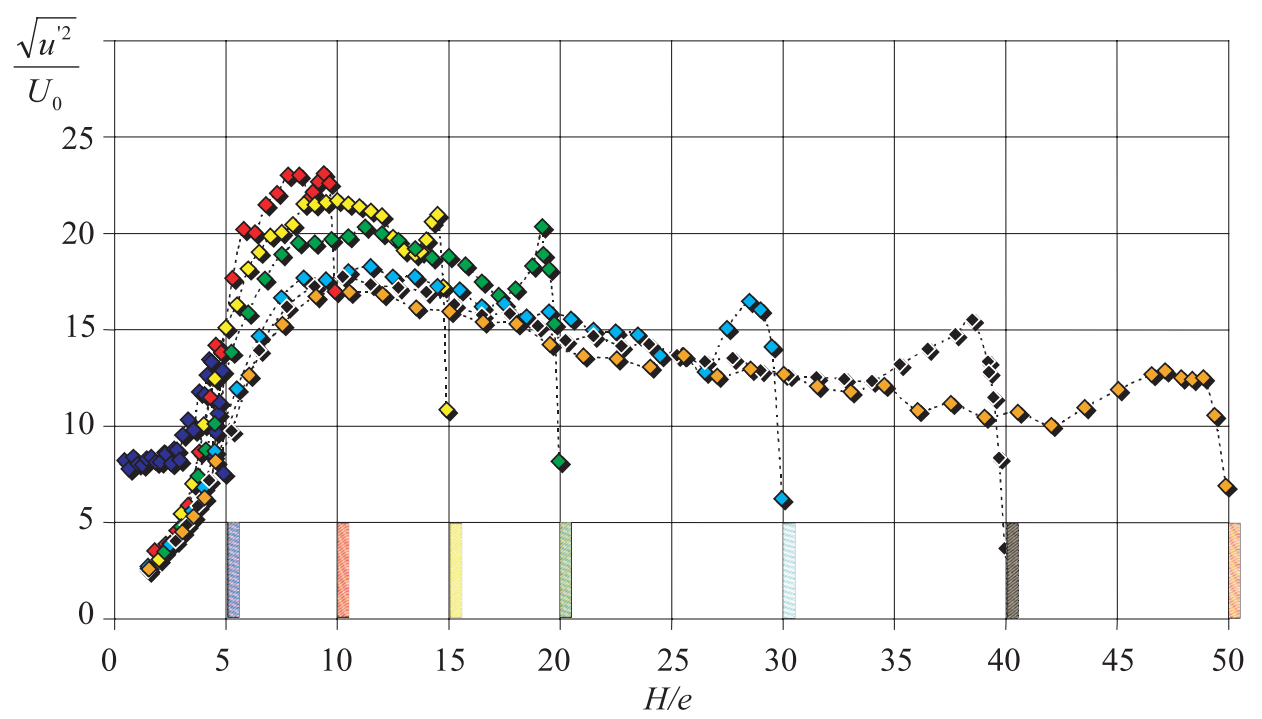

Fig. 13. Influence du rapport $H / e$ sur l'intensité de la turbulence $I_{U}$ suivant l'axe du jet, $e=20 \mathrm{~mm}, R e=27000$.

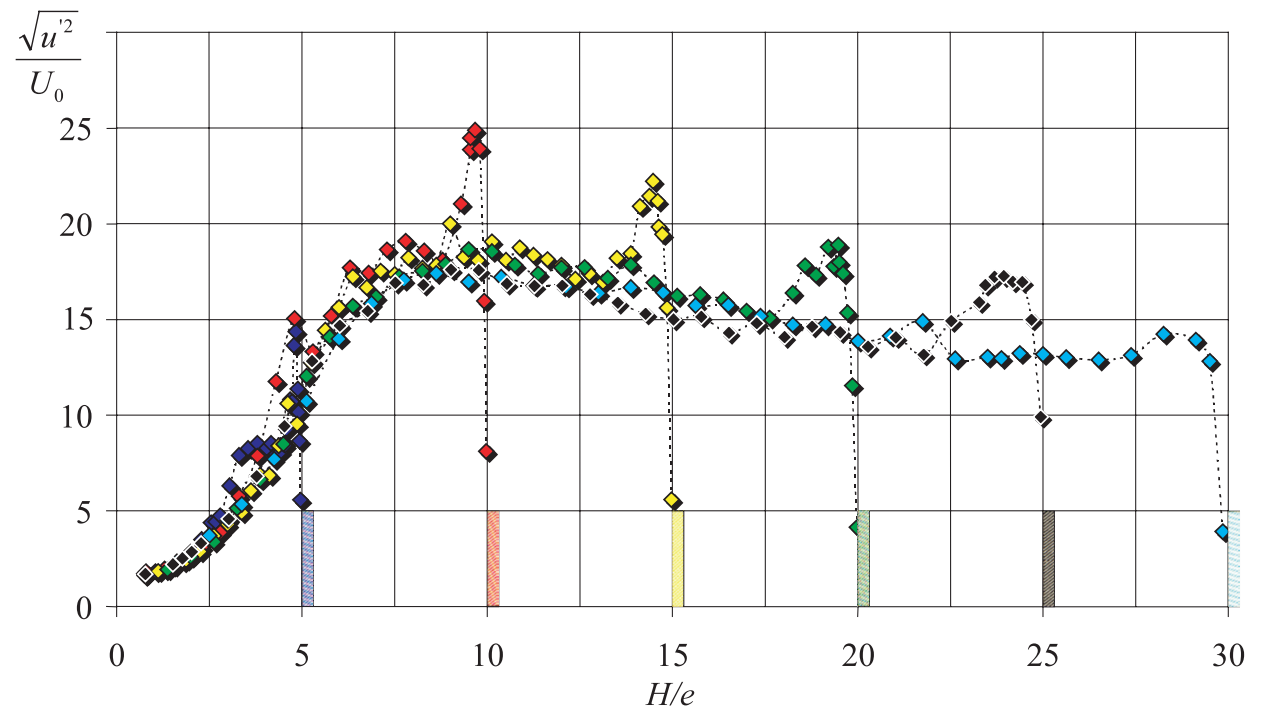

Fig. 14. Influence du rapport $H / e$ sur l'intensité de la turbulence $I_{U}$, suivant l'axe du jet, $e=40 \mathrm{~mm}, R e=27000$.

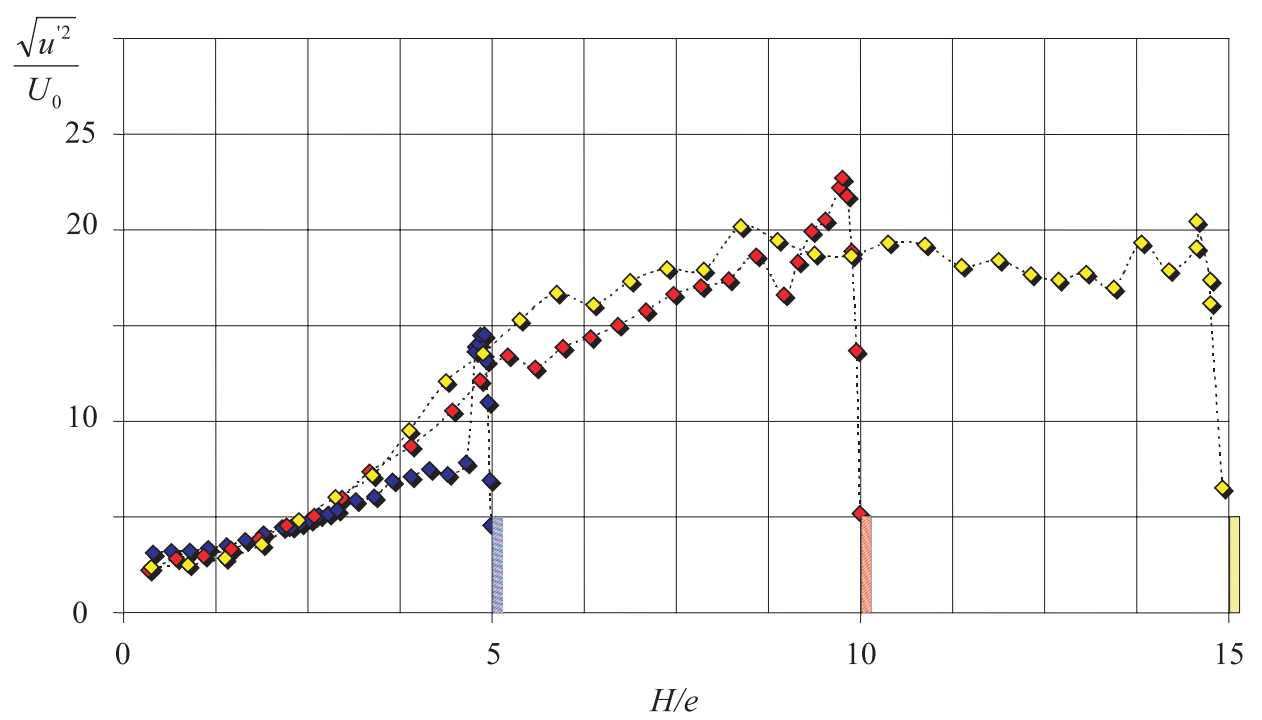

Fig. 15. Influence du rapport $H / e$ sur l'intensité de la turbulence $I_{U}$, suivant l'axe du jet, $e=80 \mathrm{~mm}, R e=27000$. 


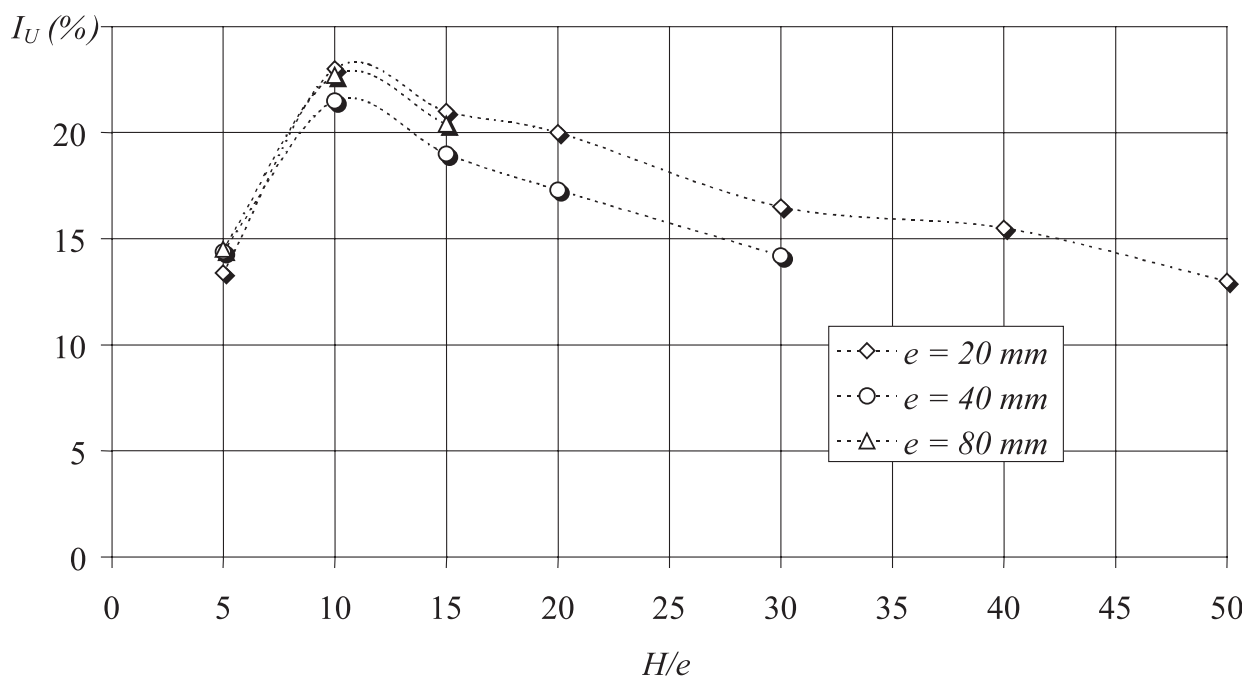

Fig. 16. Évolution du pic d'intensité de la turbulence dans la zone d'impact, $R e=27000$.

l'application, confinement ou chauffage/refroidissement de paroi, il sera important d'éviter ou de choisir cette configuration.

La demi-largeur du jet présente, quant à elle, une évolution presque linéaire en fonction du rapport $H / e$.

\section{Références}

[1] F. Beaubert, S. Viazzo, Étude d'un jet plan turbulent en impact proche par simulation des grandes échelles, Journée SFT, « Transferts de chaleur et de masse dans les jets », Paris, France, 2001

[2] C. Inard, P. Depecker, J.J. Roux, Un modèle simplifié pour la prédiction du champ de température dans les bâtiments, Rev. Gén. Therm. 36 (1997) 113-123

[3] Abramovitch, The theory of turbulent jets, Massachusetts MIT Press, 1963

[4] S. Beltaos, N. Rajaratnam, Plane turbulent impinging jets, J. Hydraulic Research 1 (1973) 29-60

[5] M. Rajaratnam, Turbulent jets, Elsevier Scientific Publishing Company, 1976

[6] R.A. Antonia, L.W. Browne, S. Rajagopalan, A.J. Chambers, On the organised motion of a turbulent plane jet, J. Fluid Mech. 134 (1983) 49-66

[7] J. Lepicovsky, Laser velocimeter measurements of largescale structures in a tone-excited jet, AIAA Journal 24 (1986) 27-31

[8] I. Namer, M.V. Ötügen, Velocity measurements in plane turbulent air jet at moderate numbers, Experiments in Fluids 6 (1988) 387-399

[9] J.M. Kuhlman, Survey of nearfield Reynolds number effects and initial condition effects on buoyant and nonbuoyant jets, ASME paper, Int. Symp. On Modelling Environmental Flows, Albuquerque/NM, USA, 1985, pp. $24-26$

[10] W.G. Jr. Hill, R.C. Jenkins, B.L. Gilbert, Effect of the initial boundary layer state on turbulent jet mixing, AIAA Journal 17 (1976) 1055-1060

[11] N.Q. Van, R.H. Howell, Influence of the initial turbulence intensity on the development of plane air-curtain jets, ASHRAE Transactions, No. 2396, 1976
[12] M. Sunyach, J. Mathieu, Zone de mélange d'un jet plan - fluctuations induites dans le cône à potentielintermittence, J. Int. Heat Mass Trans. 12 (1969) 1679-1697

[13] F.O. Thomas, V.W. Goldschmidt, Structural characteristics of a developing turbulent planar jet, J. Fluid Mech. 163 (1986) 227-256

[14] S.V. Garimella, B. Nenaydykh, Nozzle-geometry effects in liquid impingement heat transfer, Int. J. Heat Mass Trans. 14 (1996) 2915-2923

[15] S. Ashforth-Frost, K. Jambunathan, Effect of nozzle geometry and semi-confinement on the potential core of the turbulent axisymmetric free jet, Int. Com. Heat Mass Trans. 23 (1996) 155-162

[16] H. Schlichting, Boundary Layer Theory, Co. McGraw-Hill Book, New York, 6th Ed., 1968

[17] L. Guyonnaud, Étude expérimentale de rideaux d'air - Contribution à leur modélisation, thèse de doctorat, École des mines de Nantes, École centrale de Nantes, 1998

[18] P.E. Jenkins, V.W. Goldschmidt, Mean temperature and velocity in a plane turbulent jet, Trans. ASME, J. Fluid Eng. 95 (1973) 581-584

[19] J. Bashir, M.S. Uberoi, Experiments on turbulent structure and heat transfer in a two-dimensional jet, The Physics of Fluid 18 (1975) 405-410

[20] E. Gutmark, M. Wolfshtein, I. Wygnanski, The plane turbulent impinging jet, J. Fluid Mech. 88 (1978) $737-756$

[21] I. Tani, Y. Komatsu, Impingement of round jet on a flat surface, 11th Int. Congress of Applied Mechanics, Munich, Germany, 1964, pp. 672-676

[22] J. Mathieu, Contribution à l'étude aérothermique d'un jet plan évoluant en présence d'une paroi, Doctor of Science Thesis, University of Grenoble, France, 1959

[23] J. Sakakibara, K. Hishida, M. Maeda, Vortex structure and heat transfer in the stagnation region of an impinging plane jet (simultaneous measurements velocity and temperature fields by digital particle image velocimetry and laser-induced fluorescence), Int. J. Heat Mass Trans 40 (1997) 3163-3176 